\title{
Modelling and control of the flame temperature distribution using probability density function shaping Xubin Sun ${ }^{1}$, Hong Yue ${ }^{2}$ and Hong Wang ${ }^{2}$
}

${ }^{1}$ Institute of Automation, Chinese Academy of Sciences, Beijing 100080, P.R. China

${ }^{2}$ Control Systems Centre, University of Manchester, Manchester M60 1QD, U.K.

This paper presents three control algorithms for the output probability density function (PDF) control of the 2D and 3D flame distribution systems. For the 2D flame distribution systems, control methods for both static and dynamic flame systems are presented, where at first the temperature distribution of the gas jet flames along the cross-section is approximated. Then the flame energy distribution (FED) is obtained as the output to be controlled by using a B-spline expansion technique. The general static output PDF control algorithm is used in the 2D static flame system, where the dynamic system consists of a static temperature model of gas jet flames and a second-order actuator. This leads to a second-order closed-loop system, where a singular state space model is used to describe the dynamics with the weights of the B-spline functions as the state variables. Finally, a predictive control algorithm is designed for such an output PDF system. For the 3D flame distribution systems, all the temperature values of the flames are firstly mapped into one temperature plane, and the shape of the temperature distribution on this plane can then be controlled by the 3D flame control method proposed in this paper. Three cases are studied for the proposed control methods and desired simulation results have been obtained.

Key words: B-spline expansion and model predictive control; flame temperature distribution probability density function (PDF).

Address for correspondence: Xubin Sun, Institute of Automation, Chinese Academy of Sciences, Beijing 100080, P.R. China, E-mail: xubin.sun@ia.ac.cn

Hong Wang is also affiliated with Northeastern University as a Changjiang Professor, P.R. China

(C) 2006 The Institute of Measurement and Control

$10.1177 / 0142331206073124$ 


\section{Nomenclature}

$\begin{array}{ll}r & \text { Horizontal abscissa } \\ x & \text { Vertical ordinate } \\ u & \text { Vertical velocity } \\ v & \text { Horizontal velocity } \\ T & \text { Temperature } \\ T_{0} & \text { Temperature at the nozzle } \\ T_{\infty} & \text { Temperature at } \infty \text { (temperature in the environment) } \\ T_{m} & \text { Temperature at the symmetric axis } \\ \gamma & \text { Kinematical coefficient of the viscosity of the laminar flow } \\ c_{p} & \text { Specific heat capacity of the fuel } \\ \sigma_{i} & \text { Mass fraction for component } i, \Sigma \sigma_{i}=1 \\ \rho & \Sigma \rho_{i}, \text { Density of the mixture } \\ r_{i} & \text { Reaction heat of the component } i \\ b(x) & \text { Flame boundary } \\ R e_{0} & \text { The Renault number at the nozzle } \\ u_{0} & \text { The fuel jet velocity at the nozzle } \\ d_{0} & \text { Nozzle diameter } \\ \gamma\left(x, u_{0}\right) & \text { PDF, } \gamma\left(x, r, u_{0}\right) \text { for the } 3 \mathrm{D} \text { flame } \\ B_{i}(\mathrm{x}) & \text { The ith basis function } \\ C(x) & \text { Matrix made up of B-spline basis functions } \\ V\left(u_{0}\right) & \text { Weights vector of the B-spline functions } \\ J & \text { Performance function } \\ g(x) & \text { The given target PDF for the 3D flame }\end{array}$

\section{Introduction}

To improve the efficiency and safety of various boilers used in industries, the flame temperature distribution (FTD) must be well controlled (Fu et al., 1989). Generally, the source fuels of the boiler can be either blast furnace gas or pulverized coals. For the blast furnace gas case, the flame at each burner will be a gas turbulent jet flame when the fuel jet velocity is higher than a certain threshold value, and will be a gas flamelet jet flame when the velocity is lower (Pope, 1985). However, for the pulverized coals case, the flame at each burner will be of two-phase turbulent reactive flows. This means that the efficiency of the boiler is mostly determined by the status of each burner flame, which can be controlled by dynamic FTD control methods. In this context, the flame distribution can be regarded as a probability density function (PDF), where the recently developed PDF shape control can be applied (Wang, 2000). For the gas jet flame, a static model (Fu et al., 1989) has been established and used in the 2D closed-loop control systems.

In recent years, many methods have been developed for flame control. Tao and Burkhardt (1994) designed a closed-loop control system of the gas jet flame, where some CCD cameras were used to capture the multi-spectral images that are processed to produce flame features such as brightness and height. Zhou (1996) 
realized a closed-loop control system of the boiler flame by the radiant energy obtained from the temperature of the flame. Serial control was employed to solve the large inertia problem through the radiant energy as an intermediate variable. Wang et al. (1995) introduced the concept of energy balance to realize the flame distribution control. More recently, a static closed-loop control system has been investigated in Sun and Wang (2004), where a static 2D flame temperature distribution model (Fu et al., 1989) was firstly established. Flame energy distribution (FED) was then obtained from the static flame model as the output PDF. An algorithm using the PDF control concept was designed to realize the required PDF shaping. Moreover, a predictive control algorithm for the dynamic flame distribution system was presented (Sun and Wang, 2005), where the FED was also seen as the output PDF density function. However, different from the work in Sun and Wang (2004) a second-order actuator was introduced to simulate the dynamic characteristics in the real flame systems. The FED is then approximated by the B-splines functions (Wang, 2000; Yue and Wang, 2003) whose weights are taken as state variables of the dynamic system. To represent such a dynamic system, a singular state space model (Dai, 1989) was designed for the output PDF system. Stability was analysed for such a closed-loop system with predictive PDF control. It has been shown that the boundness of the first two state variables can guarantee the stability of the closed-loop system because a singular state space model is used in this system.

The 2D flame control methods are mainly designed for an axially symmetrical 3D flame distribution system, which can be easily simplified into a 2D flame distribution model. For a normal 3D flame distribution system, the 3D flame control method can be formulated where all the temperature values in the boiler are mapped into one cross-section. In practice, the temperature image captured at the top of boiler can be seen as the mapped temperature cross-section, which can well reflect the status of the boiler. A 3D flame control method is presented in this paper, the aim is to make the temperature distribution as close as possible to the given temperature distribution. Bivariate B-splines are used in the 3D flame modelling, and the output PDF control method is presented for such a 3D model, which is followed by a case study.

\section{2D flame model representation}

\subsection{Flame model simplification}

In practice, the gas flamelet jet flame distribution is of a 3D nature, which is axially symmetrical (Fu et al., 1989). Therefore, a 2D temperature distribution model can be adopted to describe the $3 \mathrm{D}$ flame distribution as shown in Figure 1, where $d_{0}$ is the diameter of the fuel injection nozzle, $u_{0}$ is the speed of the fuel injection at the nozzle, $x$ is the vertical ordinate, $r$ is the horizontal abscissa, and $b(x)$ is the flame boundary. The two arcs are the isotherms of the jet flame.

Because of the velocity distribution, temperature and mass fraction in any cross-section is similar, a Gaussian type function in Figure 2 can be used to describe such a distribution, leading to the following temperature curve 


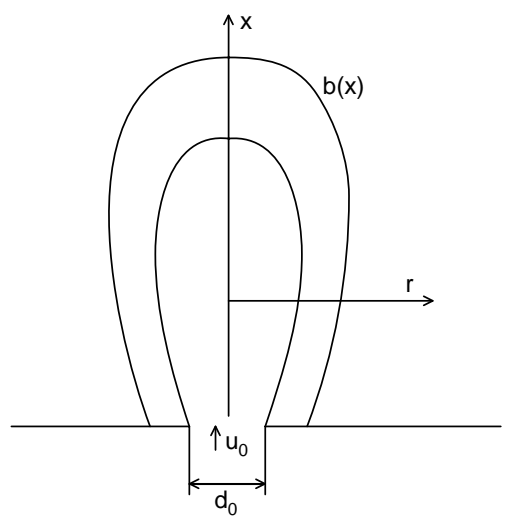

Figure 1 FTD demonstration

$$
\frac{T}{T_{m}}=\exp \left[-K\left(\frac{r}{x}\right)^{2}\right]
$$

where $T$ is the temperature at the point $(x, r), T_{m}$ is the temperature at the corresponding point on the axis $(x, 0)$ and $K$ is a coefficient with a value between 82.0 and 92.0, which is obtained from an experimental setup. To simplify the model, the following double biases distribution is used to approximate the above Gaussian distribution

$$
\frac{T}{T_{m}}=1-\frac{r}{b(x)} \quad|r| \leq b(x)
$$

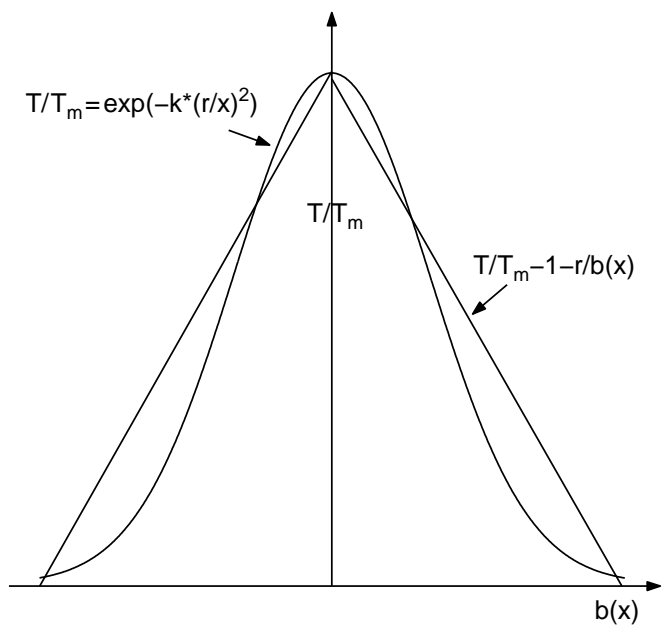

Figure 2 Temperature distribution in a cross-section before and after approximation 
A satisfactory solution can be obtained from the conservation equations after such an approximation.

\subsection{Flame model solution}

It is assumed that the flame is the uncompressible steady flow and columned free jet flame. Based upon some specific boundary and initial conditions, the FTD model can be obtained by solving the continuity, momentum, energy and mass balance conservations, leading to the following flame temperature model

$$
T(x, r)-T_{\infty}=\left[\left(T_{0}-T_{\infty}\right)-\frac{\sigma_{F} \Upsilon_{F}}{c_{p}}\right] \tau(x, r)-\frac{\sigma_{F} \Upsilon_{F}}{c_{p}}
$$

where the subscript $F$ in $\sigma_{F}$ and $Q_{F}$ stand for the fuel flows. For the flamelet flows, $\tau(x, r)$ in (3) is given by

$$
\tau(x, r)=\left(1+\frac{8}{R e_{0}} \frac{x}{d_{0}}\right)^{-1}\left[1-\sqrt{\frac{2}{3}} \frac{r}{d_{0}}\left(1+\frac{8}{R e_{0}} \frac{x}{d_{0}}\right)^{-1}\right]
$$

where $R e_{0}$ is the Renault number at the nozzle and it is defined by

$$
R e_{0}=\frac{u_{0} d_{0}}{\gamma}
$$

In (4), the input $u_{0}$ controls the value of $\tau(x, r)$. Furthermore, $u_{0}$ directly controls the temperature value of $T(x, r)$ in (3). In our case study, the parameters in the flame model have been selected as follows:

$$
\left\{\begin{array}{l}
\text { Fuel }-\mathrm{CO} \\
\left.c_{p}=2078.6 \mathrm{~J} / \mathrm{kg} \times \mathrm{K}\right) \\
\Upsilon_{F}=-282.84 \mathrm{~kJ} / \mathrm{mol} \\
d_{0}=0.01 \mathrm{~m} \\
\gamma=3.695 \times 10^{-5} \\
T_{0}=500 \mathrm{~K}
\end{array}\right.
$$

The desired FTD can be obtained though (3) and (4) with the parameters given above. The FED can then be calculated from the FTD. Physically, the flame energy is defined as the sum of all the temperature values in each horizontal cross-section, then the FED can be calculated from

$$
\gamma\left(x, u_{0}(k)\right)=\frac{\int_{0}^{+\infty} T(x, r) d r}{\int_{0}^{+\infty} \int_{0}^{+\infty} T(x, r) d r d x}
$$

By allocating different colours to represent different temperature values, a typical temperature distribution $T(x, r)$ is shown on the left-hand side of Figure 3, and its 

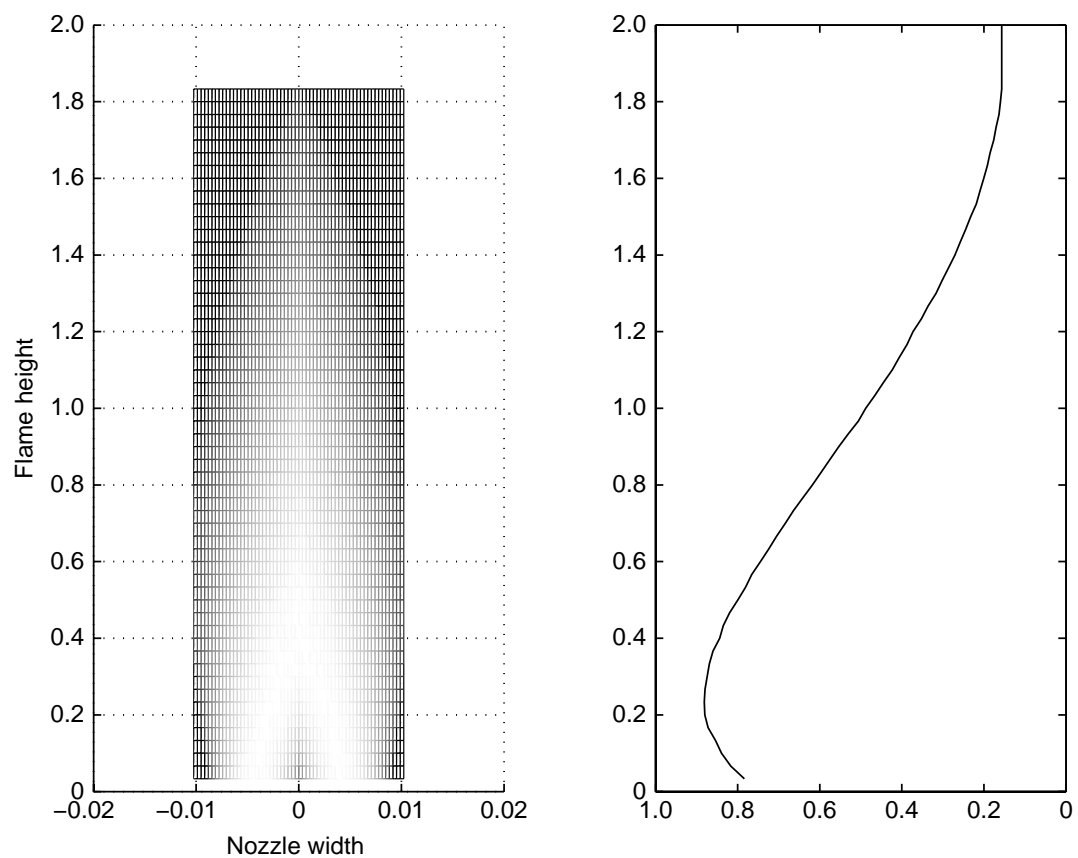

Figure 3 FTD and its corresponding FED

corresponding FED $\gamma\left(x, u_{0}(k)\right)$ is shown on the right-hand side of Figure 3 . In this case, the input is the fuel jet velocity $u_{0}(k) \in[a, b]$ where $k$ stands for the current sample time. As an FTD can be 'one-to-one' mapped into an FED, it is reasonable to control the flame distribution through the control of FEDs.

\subsection{FED modelling}

As described by Sun and Wang (2004), the FED can be approximated by the following B-splines function model

$$
\begin{gathered}
\gamma\left(x, u_{0}(k)\right)=C(x) V\left(u_{0}(k)\right)+L(x) \\
C(x)=\left[\begin{array}{l}
B_{1}(x)-\frac{B_{n+1}(x)}{\int_{a}^{b} B_{n+1}(x) d x} \int_{a}^{b} B_{1}(x) d x \\
B_{2}(x)-\frac{B_{n+1}(x)}{\int_{a}^{b} B_{n+1}(x) d x} \int_{a}^{b} B_{2}(x) d x \\
B_{n}(x)-\frac{B_{n+1}(x)}{\int_{a}^{b} B_{n+1}(x) d x} \int_{a}^{b} B_{n}(x) d x
\end{array}\right] \in R^{n \times 1}
\end{gathered}
$$


where $x \in[a, b]$ and $B_{i}(x)(i=1,2, \ldots, n+1)$ are a set of pre-specified B-spline basis functions and

$$
\begin{gathered}
V\left(u_{0}(k)\right)=\left[v_{1}\left(u_{k}\right), v_{2}\left(u_{k}\right), \ldots, v_{n}\left(u_{k}\right)\right]^{T} \in R^{n \times 1} \\
L(x)=\left(\int_{a}^{b} B_{n+1}(x) d x\right)^{-1} B_{n+1}(x) \in R^{1 \times 1}
\end{gathered}
$$

where $v_{i}\left(u_{k}\right)(i=1,2, \ldots, n)$ are the weights of the B-spline basis functions. This means that the control of the flames distribution can be realized by controlling these weights in the B-spline model.

\section{2D static system modelling and control}

The process to be controlled is the static jetting flame model as shown in (3)-(4), where the output is the FTD, which is a 2D distribution along $(x, r)$ directions (Figure 1). However, since the flame is symmetrical, another distribution, namely the FED, can be used as a feedback for the closed-loop control. In this case, the input $u_{0}(k) \in[0,2]$ is the fuel jet velocity. As the FTD can be retrieved from $\gamma\left(x, u_{0}(k)\right)$ using the symmetrical nature of the flames, the aim of the controller design is to make $\gamma\left(x, u_{0}(k)\right)$ as close as possible to a given FED. As $\gamma\left(x, u_{0}(k)\right)$ is the ratio of the energy in each cross-section to the total energy as shown in (6), it can also be regarded as a PDF, where the recently developed stochastic distribution theory (Wang, 2000) can be directly applied to control the shape of $\gamma\left(x, u_{0}(k)\right)$. A closed-loop flame distribution control system can be established as shown in Figure 4 . For this static system, the FED can be modelled using the B-splines approximation method as (7). The performance function is selected as follows

$$
J\left(u_{0}(k)\right)=\int_{0}^{2}\left(\gamma\left(x, u_{0}(k)\right)-g(x)\right)^{2} d x
$$

where $g(x)$ is the given target FED function. It can be seen that such a performance function measures the difference between the actual and the target FED distributions. Minimizing $u_{0}$ with respect to this performance function would therefore obtain the required control action that can make $\gamma\left(x, u_{0}(k)\right)$ as close as possible to $g(x)$. By substituting (7) into (11), and denoting

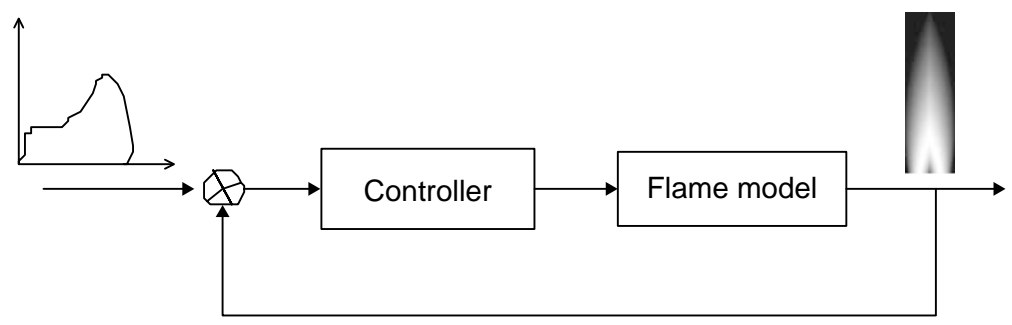

Figure 4 2D static flame control system 


$$
\begin{gathered}
\sum=\int_{0}^{2} C^{T}(x) C(x) d x \in R^{(n \times n)} \\
\eta=\int_{0}^{2}(g(x)-L(x)) C(x) d x \in R^{1 \times n} \\
\gamma_{0}=\int_{0}^{2}(g(x)-L(x))^{2} d x
\end{gathered}
$$

the control input can be calculated from the recursive form

$$
u_{0}(k)=u_{0}(k-1)-2 \mu\left(V^{T}(u) \Sigma-\eta\right) \frac{\partial V(u)}{\partial u} \mid u=u_{0}(k-1)
$$

where $\mu>0$ is a pre-specified step length. This 2D static FED control can be easily realized in practice. However, it ignores the dynamics of the whole flame system that includes the actuator and sensor dynamics, etc. As such, a more practical control method needs to be developed.

\section{Predictive control of $2 \mathrm{D}$ dynamic flame}

\subsection{Control system structure}

To consider the system dynamics, a dynamic close-loop control method is designed as shown in Figure 5. Different from the previous work in Sun and Wang (2004), a second-order actuator with a transfer function $P(s)$ is included in the system. As the flame dynamics are generally very fast, the dynamic model can reflect the actual situation in the flames control to a certain degree.

\subsection{Predictive control algorithm}

The normal state space PDF model used in Wang (2000) and Wang et al. (2005) takes the weights of the B-spline functions as its state variables and thus leads to a $n$ th-order system with the same dimension as that of $V(k)$. However, the real system in Figure 5 is of second order only. To simplify the model, a singular state space model is introduced as follows:

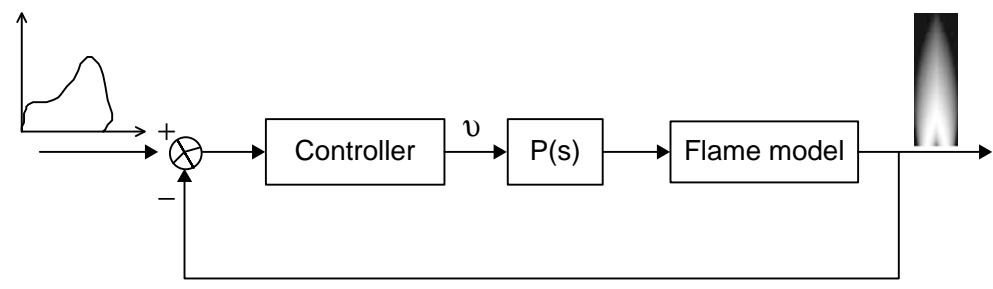

Figure 5 Dynamic closed-loop control system 


$$
\begin{aligned}
& E V(k+1)=A V(k)+B u_{0}(k) \\
& \gamma\left(x, u_{0}(k)\right)=C(x) V(k)+L(x)=f(x, k)+L(x)
\end{aligned}
$$

where $\operatorname{det}(E)=0, \operatorname{det}(A) \neq 0, x \in[a, b], E \in R^{n \times n}, \mathrm{~A} \in R^{n \times n}, \mathrm{~B} \in R^{n \times 1}$, matrices $E$ and $A$ can be selected to guarantee that only two weights in vector $V(k)$ are dynamically related to the control input, whilst the others are the linear combination of the selected two dynamical weights. Then (13) can be generally given as:

$$
\left[\begin{array}{ccccc}
1 & & & & \\
& 1 & & & \\
& & 0 & & \\
& & & \ddots & \\
& & & & 0
\end{array}\right]\left[\begin{array}{c}
v_{1}(k+1) \\
v_{2}(k+1) \\
\vdots \\
\vdots \\
v_{n}(k+1)
\end{array}\right]=\left[\begin{array}{ccccc}
a_{1} & d_{1} & 0 & 0 & 0 \\
a_{2} & d_{2} & 0 & 0 & 0 \\
a_{3} & d_{3} & -1 & 0 & 0 \\
\vdots & \vdots & 0 & \ddots & 0 \\
a_{n} & d_{n} & 0 & 0 & -1
\end{array}\right]\left[\begin{array}{c}
v_{1}(k) \\
v_{2}(k) \\
\vdots \\
\vdots \\
v_{n}(k)
\end{array}\right]+\left[\begin{array}{c}
b_{1} \\
b_{2} \\
\vdots \\
\vdots \\
b_{n}
\end{array}\right] u_{0}(k)
$$

which can be further expressed as follows:

$$
\left\{\begin{array}{l}
v_{1}(k+1)=a_{1} v_{1}(k)+d_{1} v_{2}(k)+b_{1} u_{0}(k) \\
v_{2}(k+1)=a_{2} v_{1}(k)+d_{2} v_{2}(k)+b_{2} u_{0}(k) \\
v_{3}(k)=a_{3} v_{1}(k)+d_{3} v_{2}(k)+b_{3} u_{0}(k) \\
\vdots \\
v_{n}(k)=a_{n} v_{1}(k)+d_{n} v_{2}(k)+b_{n} u_{0}(k)
\end{array}\right.
$$

The first two equations in (15) can be extracted to give

$$
\left[\begin{array}{l}
v_{1}(k+1) \\
v_{2}(k+1)
\end{array}\right]=\left[\begin{array}{ll}
a_{1} & d_{1} \\
a_{2} & d_{2}
\end{array}\right]\left[\begin{array}{l}
v_{1}(k) \\
v_{2}(k)
\end{array}\right]+\left[\begin{array}{l}
b_{1} \\
b_{2}
\end{array}\right] u_{0}(k)
$$

By taking the $z$-transforms of (16), it can be obtained that

$$
\left[\begin{array}{l}
v_{1}(k+1) \\
v_{2}(k+1)
\end{array}\right]=z^{-1}\left[\begin{array}{ll}
a_{1} & d_{1} \\
a_{2} & d_{2}
\end{array}\right]\left[\begin{array}{l}
v_{1}(k+1) \\
v_{2}(k+1)
\end{array}\right]+\left[\begin{array}{l}
b_{1} \\
b_{2}
\end{array}\right] u_{0}(k)
$$

and hence

$$
\left[\begin{array}{c}
v_{1}(k+1) \\
v_{2}(k+1)
\end{array}\right]=\left(I-z^{-1}\left[\begin{array}{ll}
a_{1} & d_{1} \\
a_{2} & d_{2}
\end{array}\right]\right)^{-1}\left[\begin{array}{l}
b_{1} \\
b_{2}
\end{array}\right] u_{0}(k)
$$

Substituting (18) into (15), the following decoupled system can be formulated:

$$
\left\{\begin{array}{l}
v_{1}(k+1)=e_{11} v_{1}(k)+e_{12} v_{1}(k-1)+e_{16} u_{0}(k)+e_{17} u_{0}(k-1) \\
v_{2}(k+1)=e_{21} v_{1}(k)+e_{22} v_{1}(k-1)+e_{26} u_{0}(k)+e_{27} u_{0}(k-1)
\end{array}\right.
$$




$$
\left\{\begin{aligned}
v_{3}(k)= & e_{31} v_{1}(k-1)+e_{32} v_{1}(k-2)+e_{33} v_{2}(k-1)+e_{34} v_{2}(k-2) \\
& +e_{35} u_{0}(k)+e_{36} u_{0}(k-1)+e_{37} u_{0}(k-2) \\
v_{4}(k)= & e_{41} v_{1}(k-1)+e_{42} v_{1}(k-2)+e_{43} v_{2}(k-1)+e_{44} v_{2}(k-2) \\
& +e_{45} u_{0}(k)+e_{46} u_{0}(k-1)+e_{47} u(k-2) \\
\vdots & \\
v_{n}(k)= & e_{n 1} v_{1}(k-1)+e_{n 2} v_{1}(k-2)+e_{n 3} v_{2}(k-1)+e_{n 4} v_{2}(k-2) \\
& +e_{n 5} u_{0}(k)+e_{n 6} u_{0}(k-1)+e_{n 7} u_{0}(k-2)
\end{aligned}\right.
$$

In the above equations, all the weights are represented by $v_{1}, v_{2}$ and $u_{0}$.

The performance function based on $\gamma(x, k)$ is used to identify the model parameters as in Wang (2000) and Wang et al. (2005). Its purpose is to make the current output $\gamma(x, k)$ and the modelled output PDF $\gamma_{g}(x, k)$ as close as possible. Such a performance function is formulated as follows

$$
J(\gamma(x, k))=\int_{a}^{b}\left(\gamma(x, k)-\gamma_{g}(x, k)\right)^{2} d x
$$

However, it is computationally difficult to estimate a large number of parameters of the singular model using any identification methods. As such, the system identification based on $v_{i}(k)$ is used in this paper and its purpose is to make each $v_{i}(k)$ and $v_{i}^{g}$, that is the $i$ th weight of the B-spline approximation, as close as possible. Such a performance function is represented as

$$
J\left(v_{i}(k)\right)=\left(v_{i}(k)-v_{i}^{g}(k)\right)^{2} \quad(i=1,2, \ldots, n)
$$

Theorem 1. When the B-spline functions are of second order, system identification based on $\gamma(x, k)$ is equivalent to the one based on the weights $v_{i}(k)$.

Proof. If the B-spline functions are of second order, then they have the following feature:

$$
\begin{gathered}
\int_{a}^{b} C_{i}(x) C_{j}(x) d x=\left\{\begin{array}{cc}
0 & |i-j|>1 \\
M_{1} \neq 0 & |i-j|=0 \\
M_{2} \neq 0 & |i-j|=1
\end{array}\right. \\
i=1,2, \ldots, n ; j=1,2, \ldots, n
\end{gathered}
$$

where $M_{1}$ and $M_{2}$ are non-zero constants.

Compare the performance function $J(\gamma(x, k))$ of the identification method based on $\gamma(x, k)$ and the performance function $J\left(v_{i}(k)\right) i=1,2, \ldots, n$ based on $v_{i}(k)$ by using the following inequality

$$
\begin{aligned}
J(\gamma(x, k)) & =\int_{a}^{b}\left(\gamma(x, k)-\gamma_{g}(x, k)\right)^{2} d x \\
& =\int_{a}^{b}\left(C(x) V(k)-C(x) V_{g}(k)\right)^{2} d x
\end{aligned}
$$




$$
\begin{aligned}
= & \int_{a}^{b} C_{1}^{2}\left(v_{1}-v_{1}^{g}\right)^{2} d x+2 \int_{a}^{b} C_{1} C_{2}\left(v_{1}-v_{1}^{g}\right)\left(v_{2}-v_{2}^{g}\right) d x \\
& +\int_{a}^{b} C_{2}^{2}\left(v_{2}-v_{2}^{g}\right)^{2} d x+2 \int_{a}^{b} C_{n-1} C_{n}\left(v_{n-1}-v_{n-1}^{g}\right)\left(v_{n}-v_{n}^{g}\right) d x \\
& +\ldots+\int_{a}^{b} C_{n}^{2}\left(v_{n}-v_{n}^{g}\right)^{2} d x
\end{aligned}
$$

According to the basic inequality $2 x y \leq x^{2}+y^{2}$, this equation can be rewritten by the following inequality that shows the relation between the performance function based on $\gamma(x, k)$ and the performance function based on $v_{i}(k)$.

$$
\begin{aligned}
J(\gamma(x, k) \leq & 2 \int_{a}^{b} C_{1}^{2}\left(v_{1}-v_{1}^{g}\right)^{2} d x+3 \int_{a}^{b} C_{2}^{2}\left(v_{2}-v_{2}^{g}\right)^{2} d x \\
& \left.+\cdots+3 \int_{a}^{b} C_{n-1}^{2}\left(v_{n-1}-v_{n-1}^{g}\right)\right)^{2} d x+2 \int_{a}^{b} C_{n}^{2}\left(v_{n}-v_{n}^{g}\right)^{2} d x \\
= & 2 M_{1}\left(v_{1}-v_{1}^{g}\right)^{2}+3 M_{1}\left(v_{2}-v_{2}^{g}\right)^{2} \\
& +\cdots+3 M_{1}\left(v_{n-1}-v_{n-1}^{g}\right)^{2}+2 M_{1}\left(v_{n}-v_{n}^{g}\right)^{2} \\
\leq & 3 n M_{1} \max _{i}\left(v_{i}-v_{i}^{g}\right)^{2}=3 n M_{1} \max _{i} J\left(v_{i}(k)\right)
\end{aligned}
$$

If $\max J\left(v_{i}(k)\right) \rightarrow 0$ can be guaranteed, then $J(\gamma(x, k)) \rightarrow 0$ will be hold. Q.E.D.

Based on this theorem, the weights equations in (19) and (20) can be identified separately. Then $f(x, k-1)$ can be described using historical information of $v_{1}, v_{2}$ and $u_{0}$ as follows:

$$
\begin{aligned}
f(x, k-1)= & C(x) V(k-1)=\sum_{i=1}^{n} C_{i}(x) v_{i}=v_{1}(k-2) \sum_{i=1, i \neq 2}^{n} e_{i 1} C_{i}(x) \\
& +v_{1}(k-3) \sum_{i=1, i \neq 2}^{n} e_{i 2} C_{i}(x)+v_{2}(k-2) \sum_{i=2}^{n} e_{i 3} C_{i}(x) \\
& +v_{2}(k-3) \sum_{i=2}^{n} e_{i 4} C_{i}(x)+u_{0}(k-1) \sum_{i=3}^{n} e_{i 5} C_{i}(x) \\
& +u_{0}(k-2) \sum_{i=1}^{n} e_{i 6} C_{i}(x)+u_{0}(k-3) \sum_{i=1}^{n} e_{i 7} C_{i}(x) \\
= & C_{1}^{\Sigma}(x) v_{1}(k-2)+C_{2}^{\Sigma}(x) v_{1}(k-3)+C_{3}^{\Sigma}(x) v_{2}(k-2) \\
& +C_{4}^{\Sigma}(x) v_{2}(k-3)+C_{5}^{\Sigma}(x) u_{0}(k-1)+C_{6}^{\Sigma}(x) u_{0}(k-2) \\
& +C_{7}^{\Sigma}(x) u_{0}(k-3)
\end{aligned}
$$

where $C_{j}^{\Sigma}(x)=\Sigma_{i=1}^{n} e_{i j} C_{i}(x)(j=1,2, \ldots, 7)$. As a result, the predicted outputs at time $k, k+1, \ldots, k+N_{p}-1$ are given by: 


$$
\begin{aligned}
f(x, k)= & \bar{f}_{11} v_{1}(k-1)+\bar{f}_{12} v_{1}(k-2)+\bar{f}_{13} v_{2}(k-1) \\
& +\bar{f}_{14} v_{2}(k-2)+q u_{0}(k-2)+h_{1} u_{0}(k-1)+\bar{g}_{11} u_{0}(k) \\
f(x, k+1 \mid k)= & f_{21} v_{1}(k)+f_{22} v_{1}(k-1)+f_{23} v_{2}(k) \\
& +f_{24} v_{2}(k-1)+h_{2} u_{0}(k-1)+\bar{g}_{21} u_{0}(k)+\bar{g}_{22} u_{0}(k+1) \\
f(x, k+2 \mid k)= & f_{31} v_{1}(k)+f_{32} v_{1}(k-1)+f_{33} v_{2}(k)+f_{34} v_{2}(k-1) \\
& +h_{3} u_{0}(k-1)+\bar{g}_{31} u_{0}(k)+\bar{g}_{32} u_{0}(k+1)+\bar{g}_{33} u_{0}(k+2) \\
& \vdots \\
f\left(x, k+N_{p}-1 \mid k\right)= & f_{N p, 1} v_{1}(k)+f_{N p, 2} v_{1}(k-1)+f_{N p, 3} v_{2}(k)+f_{N p, 4} v_{2}(k-1) \\
& +h_{N p} u_{0}(k-1)+\bar{g}_{N p, 1} u_{0}(k)+\cdots+\bar{g}_{N p, N p} u_{0}\left(k+N_{p}-1\right)
\end{aligned}
$$

where $N_{p}$ is the predictive horizon. Define vector $Y$ and matrix $F$ as follows:

$$
\begin{gathered}
Y=\left[f(x, k), f(x, k+1 \mid k), \ldots, f\left(x, k+N_{p}-1 \mid k\right)\right]^{T} \\
F=\left[\begin{array}{cccc}
f_{11} & f_{12} & f_{13} & f_{14} \\
f_{21} & f_{22} & f_{23} & f_{24} \\
\vdots & \vdots & \vdots & \vdots \\
f_{N p, 1} & f_{N p, 2} & f_{N p, 3} & f_{N p, 4}
\end{array}\right]
\end{gathered}
$$

Based upon the predictive model (23), the parameters in matrix $F$ can be obtained recursively. The first row in matrix $F$ is given as follows:

$$
\left[\begin{array}{llll}
f_{11} & f_{12} & f_{13} & f_{14}
\end{array}\right]=\left[\begin{array}{llll}
0 & C_{1}^{\Sigma} & 0 & C_{3}^{\Sigma}
\end{array}\right]
$$

and the $i$ th row in matrix $F$ can be expressed as

$$
\left[\begin{array}{llll}
f_{i 1} & f_{i 2} & f_{i 3} & f_{i 4}
\end{array}\right]=\left[\begin{array}{llll}
C_{1}^{\Sigma} & C_{2}^{\Sigma} & C_{3}^{\Sigma} & C_{4}^{\Sigma}
\end{array}\right] \times\left[\begin{array}{cccc}
e_{11} & e_{12} & 0 & 0 \\
1 & 0 & 0 & 0 \\
0 & 0 & e_{23} & e_{24} \\
0 & 0 & 1 & 0
\end{array}\right]^{i-2} \quad\left(2 \leq i \leq N_{p}\right)
$$

Define vector $Q$ which includes the information of the past time $k-2$ by

$$
\begin{aligned}
Q & =\left[\begin{array}{c}
q u_{0}(k-2)+C_{2}^{\Sigma} v_{1}(k-2)+C_{4}^{\Sigma} v_{2}(k-2) \\
0 \\
\vdots \\
0
\end{array}\right] \\
& =\left[\begin{array}{c}
C_{7}^{\Sigma} u_{0}(k-2)+C_{2}^{\Sigma} v_{1}(k-2)+C_{4}^{\Sigma} v_{2}(k-2) \\
0 \\
\vdots \\
0
\end{array}\right]
\end{aligned}
$$

Define matrix $\bar{G}$ as follows 


$$
\bar{G}=\left[\begin{array}{cccc}
\bar{g}_{11} & & & \\
\bar{g}_{21} & \bar{g}_{22} & & \\
\vdots & \vdots & \ddots & \\
\bar{g}_{N p, 1} & \bar{g}_{N p, 2} & \cdots & \bar{g}_{N p, N p}
\end{array}\right]=\left[\begin{array}{cccc}
\bar{g}_{11} & & & \\
\bar{g}_{21} & \bar{g}_{11} & & \\
\vdots & \vdots & \ddots & \\
\bar{g}_{N p, 1} & \bar{g}_{N p-1,1} & \cdots & \bar{g}_{11}
\end{array}\right]
$$

Obviously $\bar{G}$ is a lower triangle matrix, where $\bar{g}_{i, j}=\bar{g}_{i-1, j-1}(i \geq j>1)$. Moreover, the first column of matrix $\bar{G}$ is given by

$$
\left[\begin{array}{c}
C_{5}^{\Sigma} \\
C_{6}^{\Sigma} \\
C_{7}^{\Sigma}+f_{21} e_{16}+f_{23} e_{26} \\
f_{21} e_{17}+f_{31} e_{16}+f_{23} e_{27}+f_{33} e_{26} \\
\vdots \\
{\left[f_{N p-2,1} e_{17}+f_{N p-1,1} e_{16}\right.} \\
\left.+f_{N p-2,3} e_{27}+f_{N p-1,3} e_{26}\right]
\end{array}\right] \in R^{N_{p} \times 1}
$$

Define $N_{u}$ as the control horizon. As described in the general predictive control method, the input is allowed to change over the next $N_{u}$ steps. This means that $\Delta u_{0}(k+j)=0, j=N_{u}, \ldots, N_{p}-1$. As a result, $\bar{G}$ can be rewritten as follows:

$$
\begin{aligned}
& \bar{G}\left[\begin{array}{c}
u_{0}(k) \\
u_{0}(k+1) \\
\vdots \\
u_{0}\left(k+N_{u}-1\right) \\
\vdots \\
u_{0}\left(k+N_{p}-1\right)
\end{array}\right]=\bar{G}\left[\begin{array}{c}
1 \\
1 \\
1 \\
1 \\
1 \\
1
\end{array}\right] u_{0}(k-1)+\bar{G}\left[\begin{array}{cccc}
1 & & & \\
1 & 1 & & \\
\vdots & \vdots & \ddots & \\
1 & 1 & \cdots & 1 \\
\vdots & \vdots & \vdots & \vdots \\
1 & 1 & 1 & 1
\end{array}\right] \\
& \times\left[\begin{array}{c}
\Delta u_{0}(k) \\
\Delta u_{0}(k+1) \\
\vdots \\
\Delta u_{0}\left(k+N_{u}-1\right)
\end{array}\right]=\bar{G}\left[\begin{array}{c}
1 \\
1 \\
1 \\
1 \\
\vdots \\
1
\end{array}\right] u_{0}(k-1)+G\left[\begin{array}{c}
\Delta u_{0}(k) \\
\Delta u_{0}(k+1) \\
\vdots \\
\Delta u_{0}\left(k+N_{u}-1\right)
\end{array}\right]
\end{aligned}
$$

where $G$ has been defined as:

$$
G=\bar{G}\left[\begin{array}{cccc}
1 & & & \\
1 & 1 & & \\
\vdots & \vdots & \ddots & \\
1 & 1 & \cdots & 1 \\
\vdots & \vdots & \vdots & \vdots \\
1 & 1 & 1 & 1
\end{array}\right]
$$


Other vectors are defined by:

$$
\begin{aligned}
& H=\left[\begin{array}{c}
h_{1} \\
h_{2} \\
\vdots \\
h_{N p}
\end{array}\right]+\bar{G}\left[\begin{array}{c}
1 \\
1 \\
\vdots \\
1
\end{array}\right]=\left[\begin{array}{c}
C_{6}^{\Sigma} \\
C_{7}^{\Sigma} \\
f_{21} e_{17}+f_{23} e_{27} \\
f_{31} e_{17}+f_{33} e_{27} \\
\vdots \\
f_{N p-1,1} e_{17}+f_{N p-1,3} e_{27}
\end{array}\right]+\bar{G}\left[\begin{array}{c}
1 \\
1 \\
\vdots \\
1
\end{array}\right] \\
& \Delta U=\left[\Delta u_{0}(k), \Delta u_{0}(k+1), \ldots, \Delta u_{0}\left(k+N_{u}-1\right)\right]^{T} \\
& W=\left[v_{1}(k) \quad v_{1}(k-1) \quad v_{2}(k) \quad v_{2}(k-1)\right]^{T} \\
& Y_{r}(x, k)=\left[\begin{array}{llll}
f_{r}(x, k) & f_{r}(x, k+1) & \ldots & f_{r}\left(x, k+N_{p}-1\right)
\end{array}\right]^{T}
\end{aligned}
$$

where $f_{r}(x, k)$ is the given reference output. Using these vectors and matrices, equation (24) can be rewritten as:

$$
Y=G \Delta U+F W+H u_{0}(k-1)+Q
$$

The performance function that is used to obtain an optimal input value is selected in a form of an integration.

$$
J(\Delta U)=\int_{a}^{b}\left[\left(Y-Y_{r}\right)^{T}\left(Y-Y_{r}\right)+\lambda \Delta U^{T} \Delta U\right] d x
$$

The optimal input can then be obtained from the following equation:

$$
\frac{\partial J(\Delta U)}{\partial \Delta U}=\int_{a}^{b}\left\{G^{T}\left[G \Delta U+F W+H u_{0}(k-1)+Q\right]+\lambda \Delta U\right\} d x=0
$$

leading to

$$
\Delta U=\left[\int_{a}^{b} G^{T} G d x+\lambda I\right]^{-1} \times \int_{a}^{b} G^{T}\left[Y_{r}-F W-H u_{0}(k-1)-Q\right] d x
$$

Equation (28) produces an input sequence. However, only the first element of $\Delta U$ will be adopted to calculate the current input and applied to the system. This gives

$$
u_{0}(k)=u_{0}(k-1)+\Delta u_{0}(k)
$$

To realize this algorithm, the following steps should be employed:

1) Set the current sample time as $k$ and formulate the current output PDF $\gamma_{k}(x)$;

2) Use B-spline functions to estimate $\gamma_{k}(x)$, then the weights vector $V(k)$ is obtained;

3) Identify the equations using the standard recursive least-squares algorithm separately;

4) Calculate G, F, H and Q. Then calculate $\Delta U$ in (28);

5) Calculate (29) to produce the current input, increase $k$ and go back to step 1. 


\subsection{Stability analysis}

To guarantee the stability of the closed-loop control system (13) and (29), a condition needs to be established in order to guarantee the boundness and continuity of the output $\gamma\left(x, u_{0}(k)\right)$ of the closed-loop system. Obviously $C(x)$ in (13) is continuous and uniformly bounded, so the continuity and boundness of $\gamma\left(x, u_{0}(k)\right)$ can be guaranteed if the boundness of $V(k)$ can be proved when the control algorithm (29) is applied to (13). As such, the control algorithm should be transferred so as to relate $u_{0}(k)$ directly to $V(k)$. As the other weights $v_{3}(k), v_{4}(k), \ldots v_{n}(k)$ are all linear combinations of $v_{1}(k)$ and $v_{2}(k)$, the output $\gamma\left(x, u_{0}(k)\right)$ will be bounded if $v_{1}(k)$ and $v_{2}(k)$ are both bounded. As such, only the boundness of $v_{1}(k)$ and $v_{2}(k)$ needs to be considered for such a singular system.

For this purpose, the dynamic equation of $v_{1}(k)$ and $v_{2}(k)$ in (16) can be represented as follows:

$$
V_{1}(k+1)=A_{1} V_{1}(k)+B_{1} u_{0}(k)
$$

where $V_{1}(k)=\left[\begin{array}{l}v_{1}(k) \\ v_{2}(k)\end{array}\right] \in R^{2 \times 1}, A_{1}=\left[\begin{array}{ll}a_{1} & d_{1} \\ a_{2} & d_{2}\end{array}\right] \in R^{2 \times 2}, B_{1}=\left[\begin{array}{l}b_{1} \\ b_{2}\end{array}\right] \in R^{2 \times 1}$. As a result, the stability of the closed-loop system is determined by the stability of vector $V_{1}$. It is necessary to obtain a direct relationship between $V_{1}(k)$ and $u_{0}(k)$ firstly before the stability condition is analysed. For this purpose, denote the first row of the following matrix

$$
\left[\int_{a}^{b} G^{T} G d x+\lambda I\right]^{-1} \in R^{N_{u} \times N_{u}}
$$

as vector $p \in R^{1 \times N_{u}}$, then $\Delta u_{0}(k)$ can be represented as

$$
\Delta u_{0}(k)=p \int_{a}^{b} G^{T}\left[Y_{r}-F W-H u_{0}(t-1)-Q\right] d x
$$

Furthermore, vector $Q$ is divided into the following two parts:

$$
Q=Q_{u} u_{0}(k-2)+Q_{v} V_{1}(k-2)=\left[\begin{array}{c}
q \\
0 \\
\vdots \\
0
\end{array}\right] u_{0}(k-2)+\left[\begin{array}{cc}
C_{2}^{\Sigma} & C_{4}^{\Sigma} \\
0 & 0 \\
\vdots & \vdots \\
0 & 0
\end{array}\right] V_{1}(k-2)
$$

At the same time, matrix $F$ is represent as

$$
F W=F_{1} V_{1}(k)+F_{2} V_{1}(k-1)=\left[\begin{array}{cc}
f_{11} & f_{13} \\
f_{21} & f_{23} \\
\vdots & \vdots \\
f_{N_{p}, 1} & f_{N_{p}, 3}
\end{array}\right]\left[\begin{array}{l}
v_{1}(k) \\
v_{2}(k)
\end{array}\right]+\left[\begin{array}{cc}
f_{12} & f_{14} \\
f_{22} & f_{24} \\
\vdots & \vdots \\
f_{N_{p}, 2} & f_{N_{p}, 4}
\end{array}\right]\left[\begin{array}{l}
v_{1}(k-1) \\
v_{2}(k-1)
\end{array}\right]
$$

where matrix $F_{1}$, which is composed of the first and third columns of matrix $F$, contains the information at time $k$, whilst matrix $F_{2}$, which is composed of the second 
and the fourth columns of matrix $F$, groups the information up to time $k-1$. With matrices $Q_{u}, Q_{v}, F_{1}$ and $F_{2}$, the relationship between $u_{0}$ and $V_{1}$ can be obtained by rewriting Equation (29) as follows:

$$
\begin{aligned}
u_{0}(k)= & u_{0}(k-1)+\Delta u_{0}(k) \\
= & u_{0}(k-1)+p \int_{a}^{b} G^{T}\left[Y_{r}-F W-H u_{0}(t-1)-Q\right] d x \\
= & u_{0}(k-1)+p \int_{a}^{b} G^{T}\left[Y_{r}-F_{1} V_{1}(k)-F_{2} V_{1}(k-1)\right. \\
& \left.-H u_{0}(t-1)-Q_{u} u_{0}(k-2)-Q_{v} V_{1}(k-2)\right] d x
\end{aligned}
$$

The equation can be further written as

$$
\begin{array}{r}
u_{0}(k)-\left[1-p \int_{a}^{b} G^{T} H d x\right] u_{0}(k-1)+\int_{a}^{b} G^{T} Q_{u} d x u_{0}(k-2) \\
=p \int_{a}^{b} G^{T}\left[Y_{r}-F_{1} V_{1}(k)-F_{2} V_{1}(k-1)-Q_{v} V_{1}(k-2)\right] d x
\end{array}
$$

where the left-hand side of this equation consists of the information on $u_{0}$ and the right-hand side consists of the information on $V_{1}$. Taking the $z$ transforms of this equation, it can be obtained that

$$
\begin{gathered}
u_{0}(k)-\left[1-p \int_{a}^{b} G^{T} H d x\right] u_{0}(k) z^{-1}+\int_{a}^{b} G^{T} Q_{u} d x u_{0}(k) z^{-2} \\
=p \int_{a}^{b} G^{T}\left[Y_{r}-F_{1} V_{1}(k)-F_{2} V_{1}(k-1)-Q_{v} V_{1}(k-2)\right] d x
\end{gathered}
$$

which leads to the following format

$$
\left[1-\Omega_{1} z^{-1}+\Omega_{2} z^{-2}\right] u_{0}(k)=p \int_{a}^{b} G^{T}\left[Y_{r}-F_{1} V_{1}(k)-F_{2} V_{1}(k-1)-Q_{v} V_{1}(k-2)\right] d x
$$

This means that $u_{0}(k)$ can be represented by the current and past information of $V_{1}$ to give

$$
u_{0}(k)=\Omega^{-1} p \int_{a}^{b} G^{T}\left[Y_{r}-F_{1} V_{1}(k)-F_{2} V_{1}(k-1)-Q_{v} V_{1}(k-2)\right] d x
$$

where $\Omega=1-\Omega_{1} z^{-1}+\Omega_{2} z^{-2}$. Substituting this equation into (30), it can be obtained that

$$
V_{1}(k+1)=A_{1} V_{1}(k)+B_{1} \Omega^{-1} p \int_{a}^{b} G^{T}\left[Y_{r}-F_{1} V_{1}(k)-F_{2} V_{1}(k-1)-Q_{v} V_{1}(k-2)\right] d x
$$

At this stage, one can multiply $\Omega$ on both sides of this equation to formulate the following 


$$
\begin{gathered}
\left(1-\Omega_{1} z^{-1}+\Omega_{2} z^{-2}\right) V_{1}(k+1)-\left(1-\Omega_{1} z^{-1}+\Omega_{2} z^{-2}\right) A_{1} V_{1}(k) \\
=B_{1} p \int_{a}^{b} G^{T}\left[Y_{r}-F_{1} V_{1}(k)-F_{2} V_{1}(k-1)-Q_{v} V_{1}(k-2)\right] d x
\end{gathered}
$$

which can be rewritten as

$$
\begin{aligned}
V_{1}(k+1)= & {\left[\Omega_{1} I+A_{1}-B_{1} p \int_{a}^{b} G^{T} F_{1} d x\right] V_{1}(k) } \\
& -\left[\Omega_{2} I+\Omega_{1} A_{1}+B_{1} p \int_{a}^{b} G^{T} F_{2} d x\right] V_{1}(k-1) \\
& +\left[\Omega_{2} A_{1}-B_{1} p \int_{a}^{b} G^{T} Q_{v} d x\right] V_{1}(k-2) \\
& +B_{1} p \int_{a}^{b} G^{T} Y_{r} d x
\end{aligned}
$$

where $I \in R^{2 \times 2}$ is a unit matrix. In the above equation the value of $V_{1}(k+1)$ is a linear combination of $V_{1}(k), V_{1}(k-1)$ and $V_{1}(k-2)$. Simplifying this equation leads to

$$
V_{1}(k+1)=\Phi_{1} V_{1}(k)+\Phi_{2} V_{1}(k-1)+\Phi_{3} V_{1}(k-2)+\Phi_{4}
$$

where

$$
\begin{aligned}
& \Phi_{1}=\Omega_{1} I+A_{1}-B_{1} p \int_{a}^{b} G^{T} F_{1} d x \in R^{2 \times 2} \\
& \Phi_{2}=-\left[\Omega_{2} I+\Omega_{1} A_{1}+B_{1} p \int_{a}^{b} G^{T} F_{2} d x\right] \in R^{2 \times 2} \\
& \Phi_{3}=\Omega_{2} A_{1}-B_{1} p \int_{a}^{b} G^{T} Q_{v} d x \in R^{2 \times 2} \\
& \Phi_{4}=B_{1} p \int_{a}^{b} G^{T} Y_{r} d x
\end{aligned}
$$

Based upon the recursive form of $V_{1}$, the value of $v_{1}(k+1)$ can be represented as follows

$$
V_{1}(k+1)=\left[\begin{array}{lll}
\Phi_{1} & \Phi_{2} & \Phi_{3}
\end{array}\right]\left[\begin{array}{ccc}
\Phi_{1} & \Phi_{2} & \Phi_{3} \\
I & 0 & 0 \\
0 & I & 0
\end{array}\right]^{k-3}\left[\begin{array}{c}
V_{1}(3) \\
V_{1}(2) \\
V_{1}(1)
\end{array}\right]+\Phi_{4} S_{q}(k-4)
$$

where $S_{q}(k-4)=[1+s(0)+s(1)+\cdots+s(k-4)]$ is a progression term whose components are determined by 


$$
s(i)=\left[\begin{array}{lll}
\Phi_{1} & \Phi_{2} & \Phi_{3}
\end{array}\right]\left[\begin{array}{ccc}
\Phi_{1} & \Phi_{2} & \Phi_{3} \\
I & 0 & 0 \\
0 & I & 0
\end{array}\right]^{i}\left[\begin{array}{l}
1 \\
0 \\
0
\end{array}\right] \quad(i \geq 0)
$$

Obviously, if the matrix $\left[\begin{array}{ccc}\Phi_{1} & \Phi_{2} & \Phi_{3} \\ I & 0 & 0 \\ 0 & I & 0\end{array}\right]$ is stable (ie, all its eigenvalues are inside the unit circle), the first term on the right-hand side of (53) will be bounded. If $k \rightarrow \infty$, then

$$
\left[\begin{array}{lll}
\Phi_{1} & \Phi_{2} & \Phi_{3}
\end{array}\right]\left[\begin{array}{ccc}
\Phi_{1} & \Phi_{2} & \Phi_{3} \\
I & 0 & 0 \\
0 & I & 0
\end{array}\right]^{k-3} \rightarrow \infty
$$

Also, if the matrix $\left[\begin{array}{ccc}\Phi_{1} & \Phi_{2} & \Phi_{3} \\ I & 0 & 0 \\ 0 & I & 0\end{array}\right]$ is stable, then the dynamic evolution will be convergent. This means that $S_{q}(k-4)<\infty$ where $k$ can be any big integer. To summarize, we have the following theorem.

Theorem 2. Suppose that $Y_{r}$ and $C(x)$ are continuous and uniformly bounded, then the closed-loop system (13) and (29) is stable if the eigenvalues of the matrix $\left[\begin{array}{ccc}\Phi_{1} & \Phi_{2} & \Phi_{3} \\ I & 0 & 0 \\ 0 & I & 0\end{array}\right]$ are all inside the unit circle.

As the dynamic term $P(s)$ is added in the control system, the flame model becomes more complex and is also closer to practical cases than that of the static model. These two control methods can be used for the flame distribution systems that can be simplified into a 2D flame distribution. For the irregular flame distributions, 3D control methods need to be designed.

\section{3D control method}

The above two flame control methods are only effective for regular flame cases, especially for axially symmetric flame distributions. To control complicated flame distributions, a 3D flame distribution control will be described in this section. Although irregular 3D flames cannot be simplified into 2D flames, it can be simplified in other ways to realize the flame shape control. Two ways can be considered, one is to select a characteristic temperature plane from the $3 \mathrm{D}$ temperature space as the output of the system, the other is to map all the temperature values into a given plane. After an appropriate normalization, the temperature distribution $\gamma\left(x, r, u_{0}(k)\right)$ can be obtained which satisfies

$$
\int \gamma\left(x, r, u_{0}(k)\right) d x d r=1
$$

where $x$ and $r$ are the ordinate axis and abscissa axis of the obtained plane. Although $\gamma\left(x, r, u_{0}(k)\right)$ is not a PDF, it can be controlled using the output PDF control method. 
For static irregular flames, $\gamma\left(x, r, u_{0}(k)\right)$ can be modelled by B-spline functions. The aim of the flame control is to make the $\gamma\left(x, r, u_{0}(k)\right)$ as close as possible to the following given temperature distribution.

$$
\gamma\left(x, r, u_{k}\right)=f\left(x, r, u_{k}\right)+L(x, r)=C(x, r) V(u(k))+L(x, r)
$$

where

$$
\begin{aligned}
& C^{T}(x, r)=\left[\begin{array}{l}
B_{1}(x, r)-\frac{B_{n}(x, r)}{\int B_{n}(x, r) d x d r} \int B_{1}(x, r) d x d r \\
B_{2}(x, r)-\frac{B_{n}(x, r)}{\int B_{n}(x, r) d x d r} \int B_{2}(x, r) d x d r \\
\vdots \\
B_{n-1}(x, r)-\frac{B_{n}(x, r)}{\int B_{n}(x, r) d x d r} \int B_{n-1}(x, r) d x d r
\end{array}\right] \\
& L(x, r)=\left(\int B_{n}(x, r) d x d r\right)^{-1} B_{n}(x, r)
\end{aligned}
$$

and $B_{i}(x, r)(i=1,2, \ldots, n)$ are a series of bivariate B-spline basis functions, which can be formed by taking a tensor product of two univariate B-splines as follows (Figure 6).

$$
B_{k, i}(x, r)=\prod_{j=1}^{2} B_{k_{j} i_{j}}(x)=B_{k_{x} i_{x}}(x) B_{k_{r} i_{r}}(x)
$$

In the above equation, $k$ represents the order of each B-spline, and $i$ stands for the $i$ th Bspline.

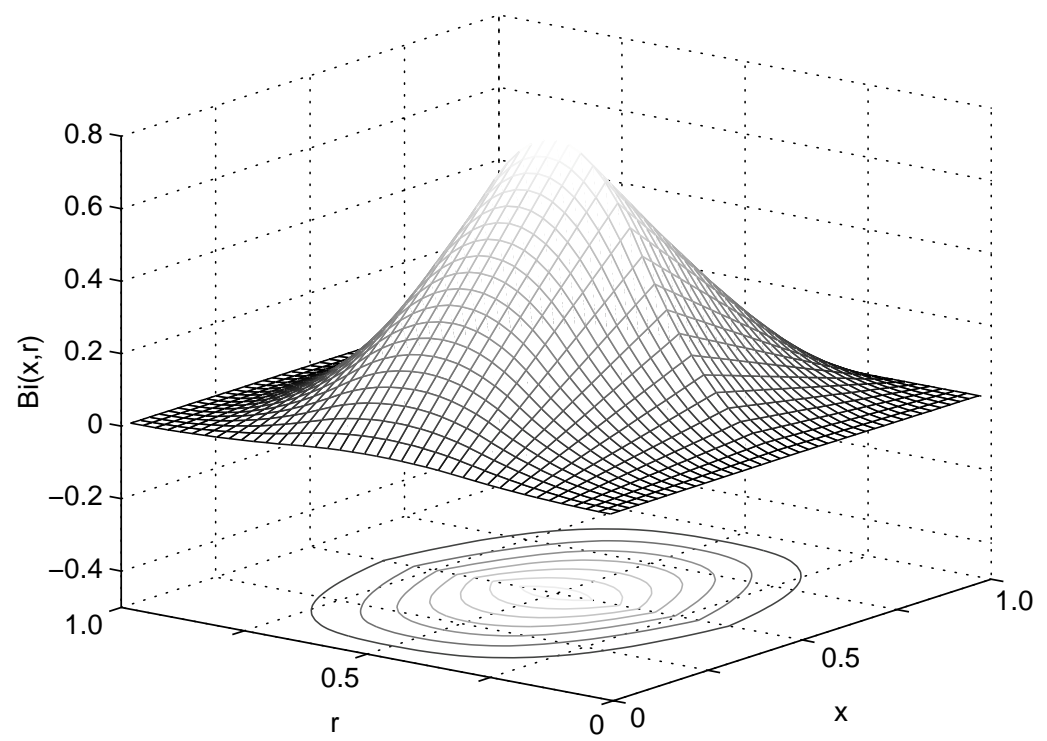

Figure 6 Tensor product of one order 2 and one order 3 univariate B-splines 


\subsection{System identification}

The discrete-time form of the system in (43) is presented when the system identification algorithm is considered. In (43), the term $L(x, r)$ is known when the Bspline functions are given. This means that only the identification of $f\left(x, r, u_{k}\right)$ should be considered.

Taking $f_{k}(i, j),\left[C_{1}(i, j), C_{2}(i, j), \ldots, C_{n}(i, j)\right] i=1,2, \ldots, n_{x}, j=1,2, \ldots, n_{r}$ in the discretetime with integers $n_{x}$ and $n_{r}$, these 2D matrices can be transferred into 1D ones to read

$$
\begin{aligned}
f_{k}\left[i \times\left(n_{x}-1\right)+j\right] & =f_{k}(i, j) \\
C_{1,2, \ldots, n-1}\left[i \times\left(n_{x}-1\right)+j\right] & =C_{1,2, \ldots, n-1}(i, j)
\end{aligned}
$$

As such, Equation (43) can be rewritten as

$$
f_{k}=C V_{k}
$$

where $f_{k}$ and $V_{k}$ are vectors and $C$ is a matrix. The standard least-square (LS) identification algorithm can then be used to produce

$$
V_{k}=\left(C^{T} C\right)^{-1} C^{T} f_{k}
$$

Other identification methods can also be used in this part.

\subsection{D static flame control algorithm}

The performance function is again selected as

$$
J=\iint\left[\gamma\left(x, r, u_{0}(k)\right)-g(x, r)\right]^{2} d x d r
$$

where $g(x, r)$ is a given temperature distribution. Substituting (43) into (46) yields

$$
J=\iint\left[C(x, r) V\left(u_{0}(k)\right)+L(x, r)-g(x, r)\right]^{2} d x d r
$$

which can be further expressed as

$$
J=V^{T}\left(u_{0}(k)\right) \sum V\left(u_{0}(k)\right)-2 \eta V\left(u_{0}(k)\right)+\gamma_{0}
$$

where it has been denoted that

$$
\begin{aligned}
\sum & =\iint\left[C^{T}(x, r) C(x, r)\right] d x d r \\
\eta & =\iint[g(x, r)-L(x, r)] C(x, r) d x d r \\
\gamma_{0} & =\iint\left[g(x, r)-L(x, r)\left[{ }^{2} d x d r\right.\right.
\end{aligned}
$$

and $u_{0}(k)$ is to be calculated to minimize $J$. This leads to the following equation 


$$
\frac{\partial J}{\partial u_{0}(k)}=0
$$

Substituting (48) into (49), it can be obtained that

$$
\left(V^{T}\left(u_{0}(k)\right) \sum-\eta\right) \frac{\partial V\left(u_{0}(k)\right)}{u_{0}(k)}=0
$$

Since the relationship between $V$ and $u_{0}$ is nonlinear, the gradient method can be used to calculate the optimal solution to give

$$
u_{0}(k)^{i+1}=u_{0}(k)^{i}-2 \mu\left(V^{T}\left(u_{0}(k)\right) \sum-\eta\right) \frac{\partial V\left(u_{0}\right)}{u_{0}} \mid u_{0}=u_{0}(k)^{i}
$$

where $\mu$ is a given step length, and $i=1,2, \ldots$

\section{Simulations and results}

\subsection{D static flame control simulation}

Forty second-order basis functions are used to approximate the FED curve. Since one input generates one unique FED curve, the target FED curve is determined by a given input $u_{g}$. Two set of simulation results are presented where the value of the step length $\mu$ in Equation (12) is determined in two different ways. In the first case, $\mu$ is set as a given constant value 0.2 . In the second case, $\mu$ is determined by the Linear Search Method of the well known Levenberg-Marquardt Nonlinear Programming in the range of $(0,0.3]$. In practice the range of $\mu$ can be obtained based upon the response speed of the system. Indeed, the value of $\mu$ determines the speed of convergence with a large $\mu$ producing a fast convergence. Figure 7 shows the responses of the control input as calculated from (12). Figure 8 gives the responses of the performance function in (11). The 3D plots in Figures 9 and 10 show how the closed-loop control can be realized so as to control the distribution of FED towards its target distribution.

In the simulation, a static model of the flame distribution system was derived and used for the closed-loop control design. Under the assumption that the flame is symmetric with respect to the vertical axis, the FED function is used to realize the control of the FTD. Since such a distribution can now be measured through a video camera and an image processing, the proposed control can be used to construct an effective closed-loop control for practical flame distribution systems.

\subsection{D dynamic flame control simulation}

In the simulation, $u_{0}$ is defined in [0,2.5] and Figure 11 (which is similar to Figure 9) shows FEDs defined on the input interval. In this case, the target FED is given by $\gamma\left(x, u_{0}^{r}\right)$, which is determined by $u_{0}^{r}$. By setting the reference input to be $u_{o}^{r}=1.9348$ and 


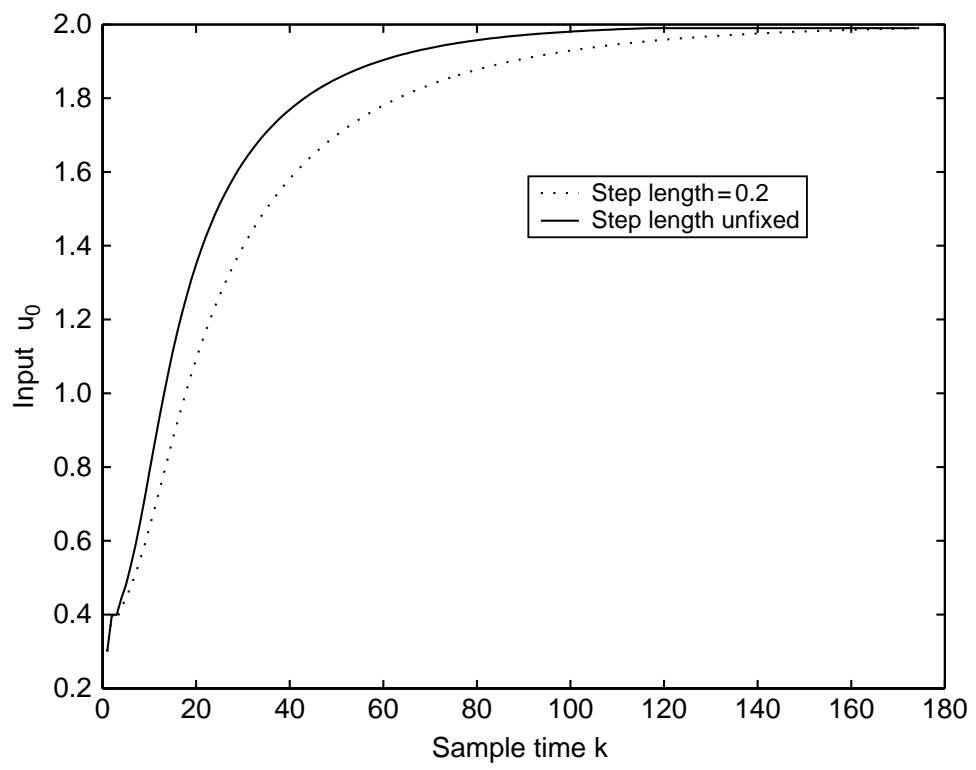

Figure 7 The responses of the control input sequences

$$
Y_{r}(x)=\left[f_{r}(x, 1.9348) \quad \ldots \quad f_{r}(x, 1.9348)\right]
$$

the control objective is to find a control rule so that the output FED follows the given FED as close as possible. A second-order B-spline with 41 basis functions is used to model the process with $u_{0}(1)=1, \lambda=2, N_{p}=4$, and $N_{u}=1,2,3$. The value of parameter

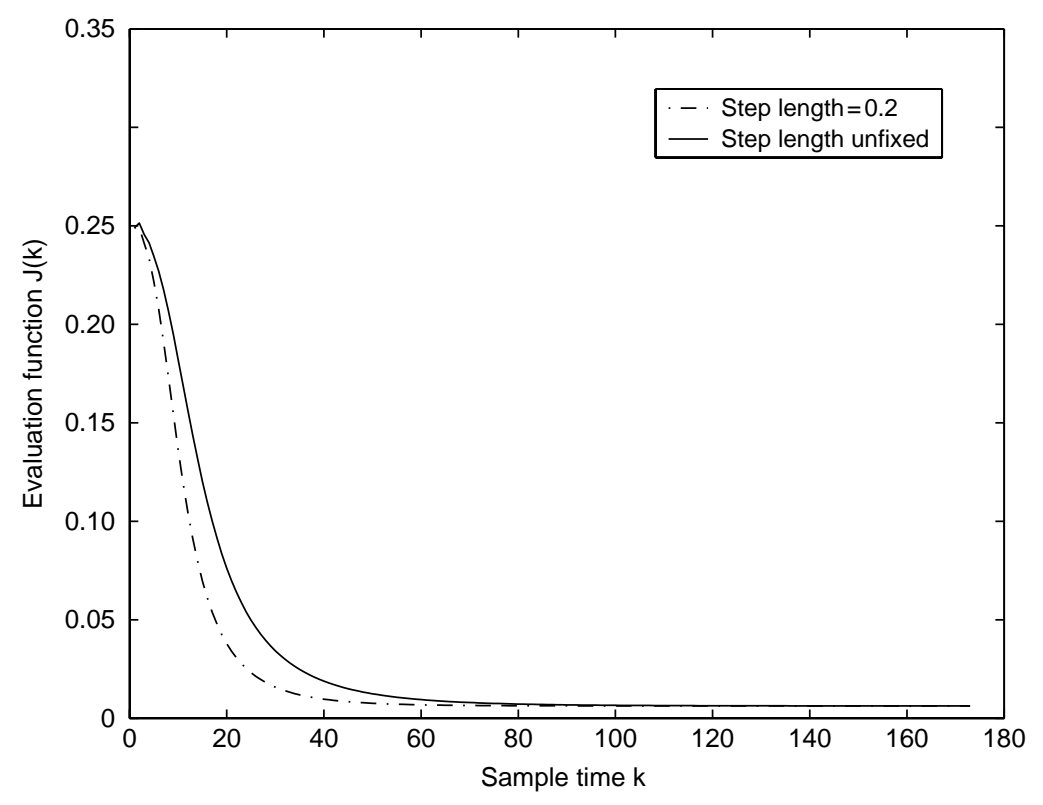

Figure 8 The responses of the evaluation functions 


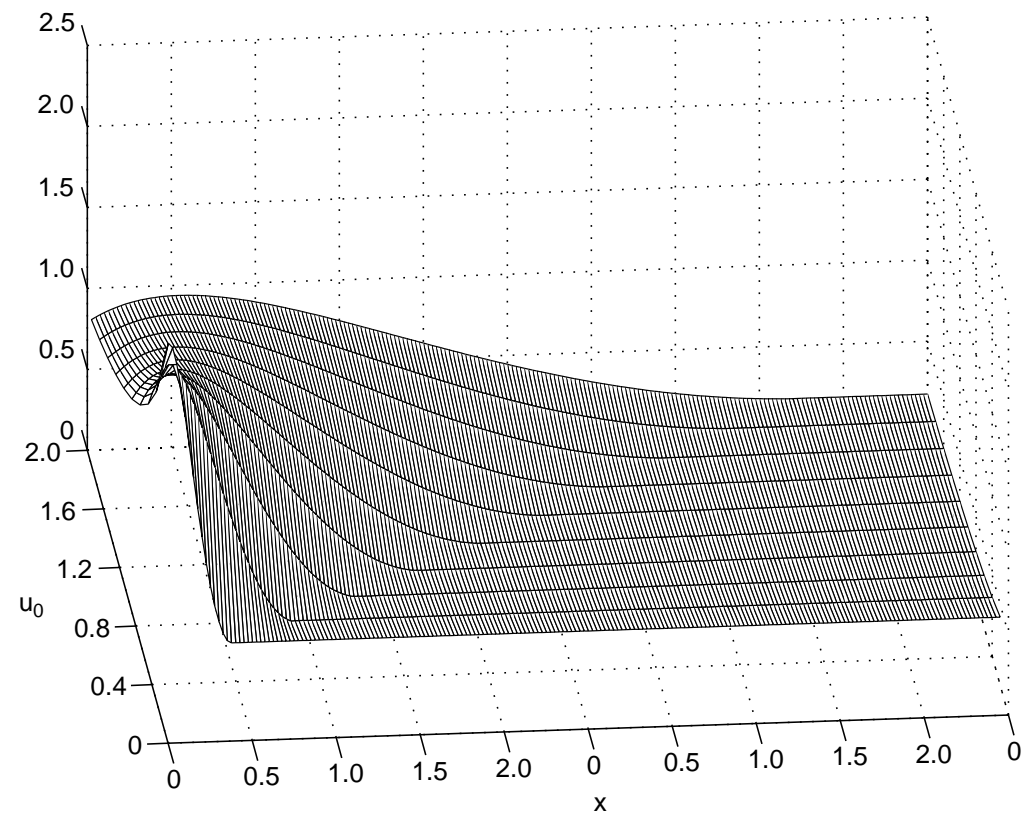

Figure 9 The closed-loop response of the FED

$\lambda$ influences the speed of the convergence and the quantity of control. In general, a large value of $\lambda$ will lead to a fast speed of the convergence. The transfer function in Figure 5 is given by

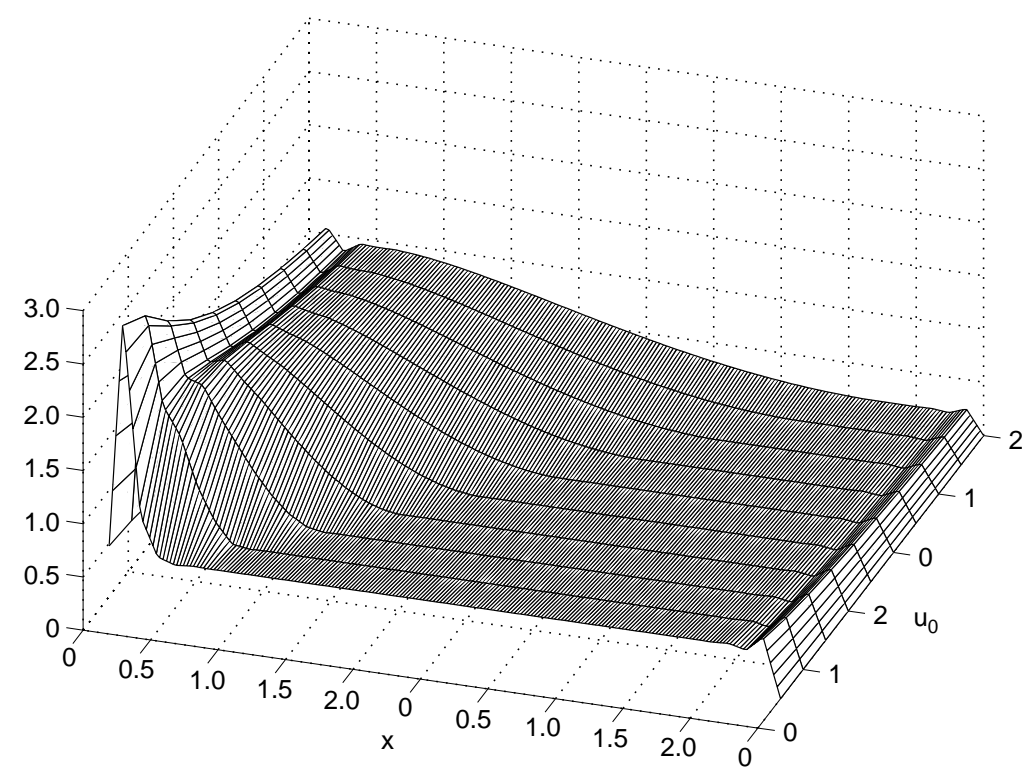

Figure 10 The estimated FED using the B-spline model 


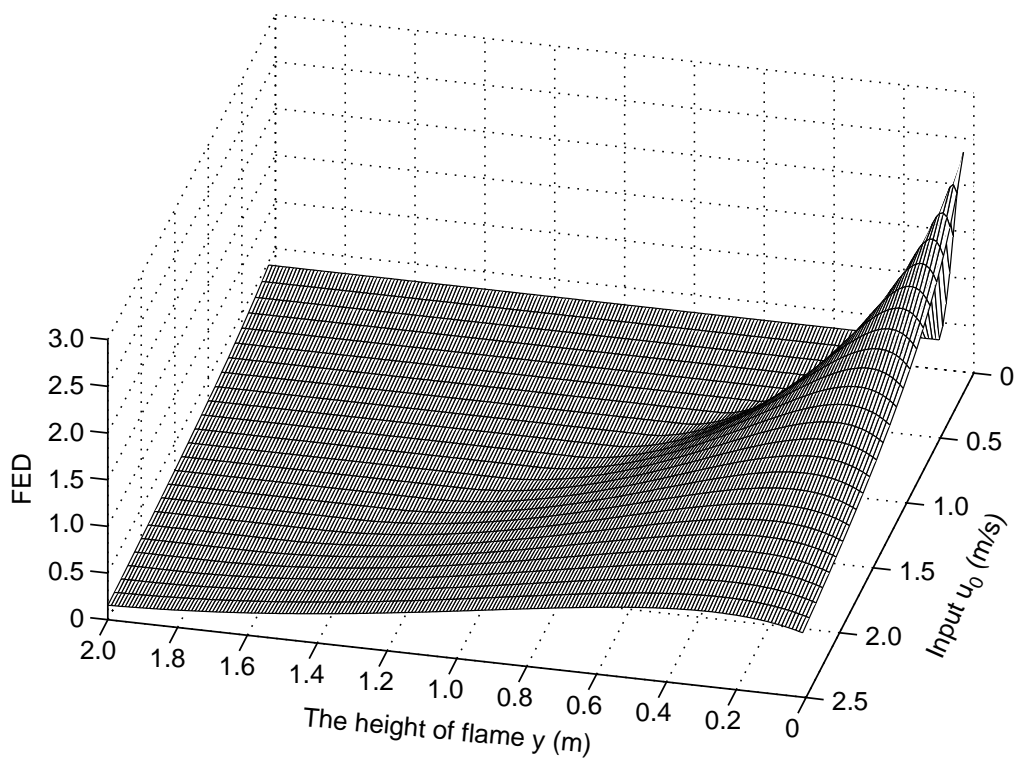

Figure 11 The given FEDs

$$
P(s)=\frac{0.8491}{s^{2}+1.6 s+1}
$$

leading to the control sequence shown in Figure 12. In Figure 12, $N_{u}=2$ gives the best control sequence when $N_{p}=4$.

\subsection{D static flame control simulation}

To test the 3D flame control algorithm, the following static flame model is used

$$
\gamma\left(x, r, u_{0}\right)=\frac{x r(x-1)(r-1)\left(x-1-u_{0}\right)\left(x-2-u_{0}\right)}{\int_{0}^{1} \int_{0}^{1} x r(x-1)(r-1)\left(x-1-u_{0}\right)\left(x-2-u_{0}\right) d x d r}
$$

This PDF $\gamma\left(x, r, u_{0}\right)$ can be seen as the temperature distribution of the image captured from the top of the boiler and $u_{0}$ can be regarded as the input and $x, r, u$ are all defined in the domain $[0,1]$. In the above equation, $u_{0}$ directly controls the shape of $\gamma\left(x, r, u_{0}\right)$. To simulate the system, let the initial PDF be determined by $u_{0}=0.95$, and the target PDF obtained by $u_{0}=0.1$. Define the step length as $\mu=0.01$, then six B-spline basis functions of the third-order for the $x$ axis and four functions of the second-order for the $r$ axis can be formed. The results are given in the Figures 13-17. 
Sun et al. $\quad 425$

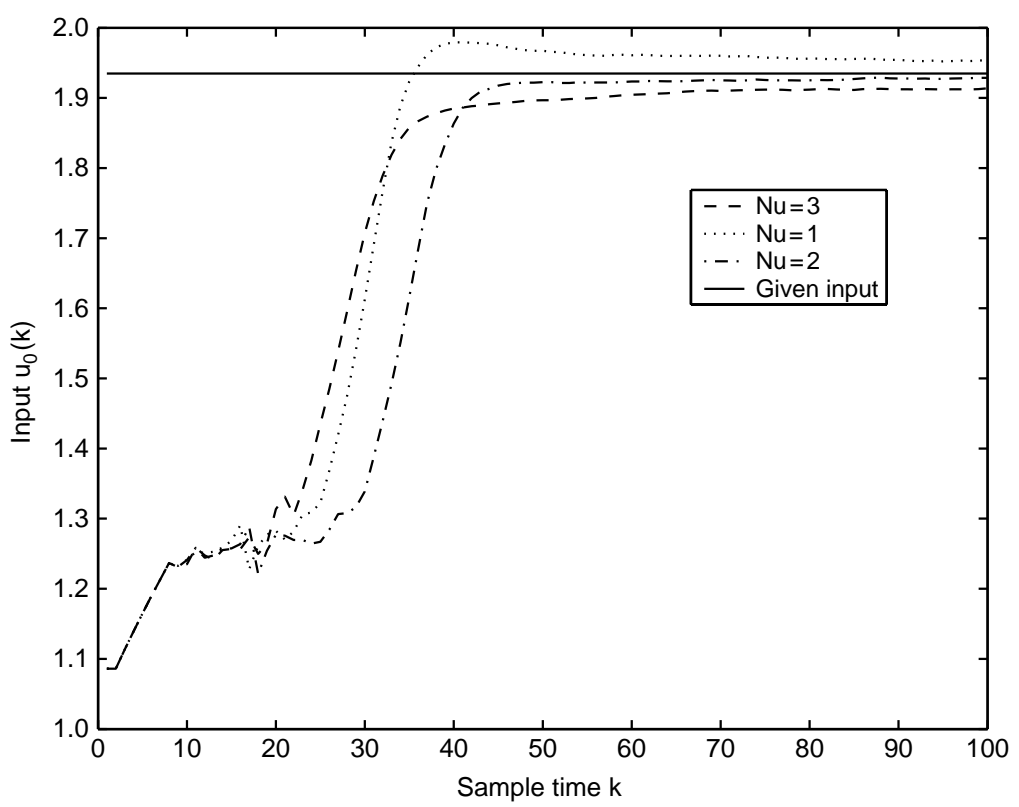

Figure 12 The 2D dynamic control sequence with different values of $N_{u}$

\section{Conclusion}

Three flame distribution control methods are presented for the FTD control in this paper via the use of stochastic distribution control ideas. The first two methods are

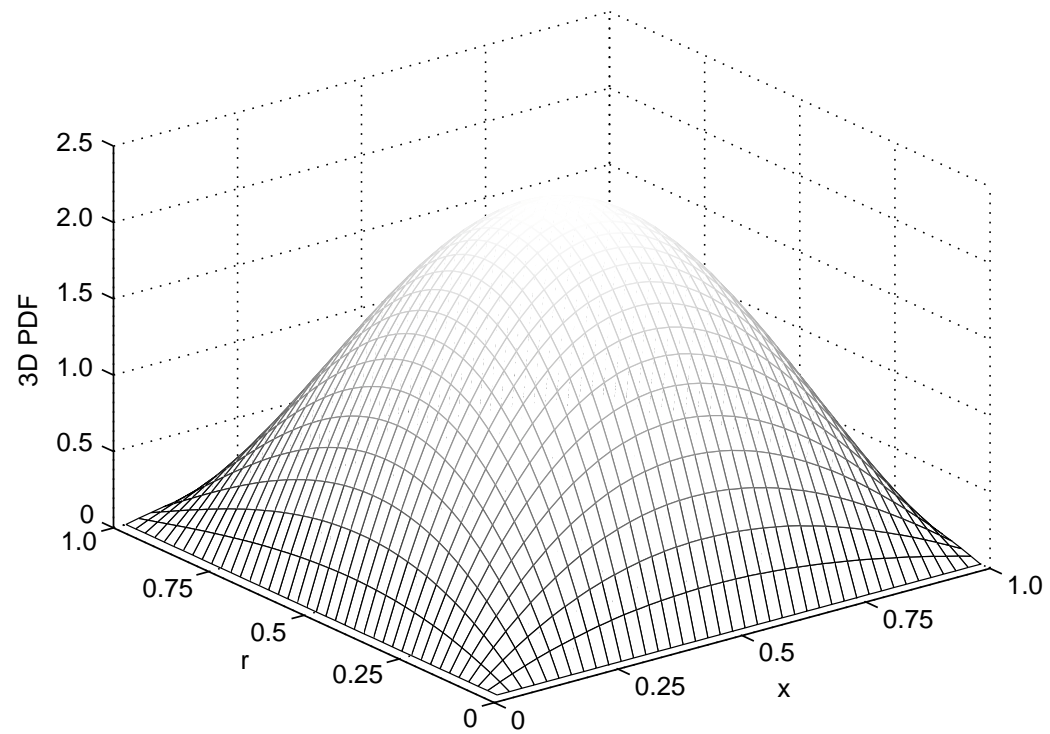

Figure 13 The initial PDF of 3D static control simulation 


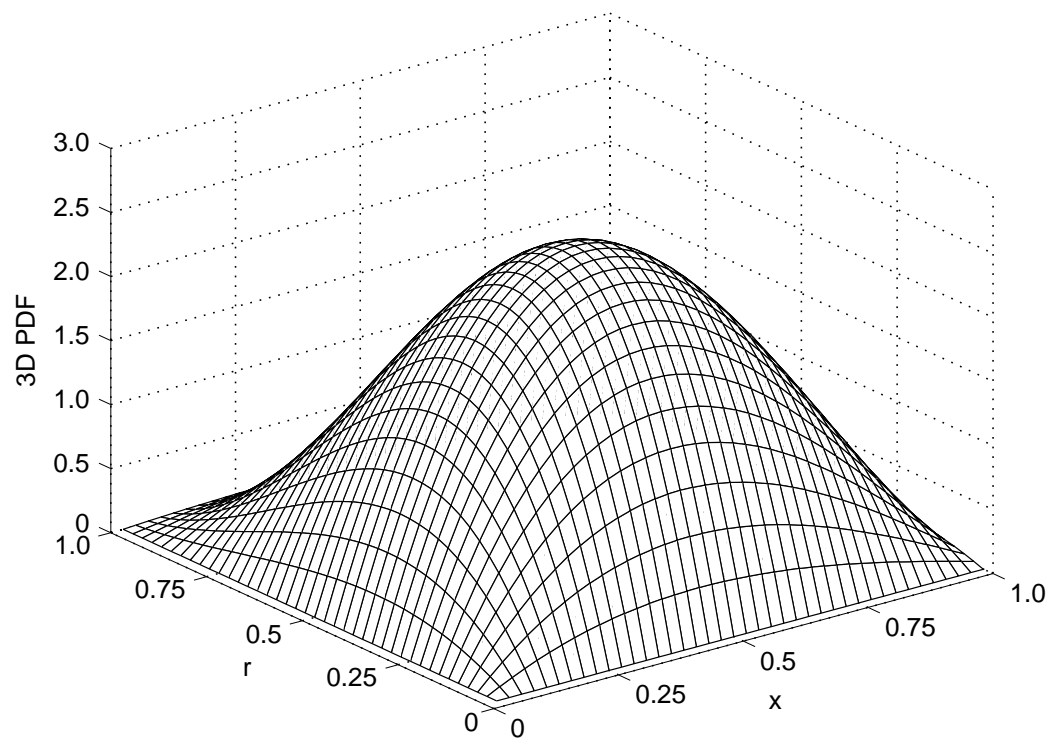

Figure 14 The given PDF of 3D static control simulation

suitable for axially symmetric flame distributions, which can be simplified into 2D flame distributions. Ignoring the system dynamics, the 2D static control can be easily realized in practice. However, the $2 \mathrm{D}$ dynamic control method is much closer to practical situations than the static ones because a dynamic term is added in the control system so as to represent the actuator effect in real systems. It is important to note that

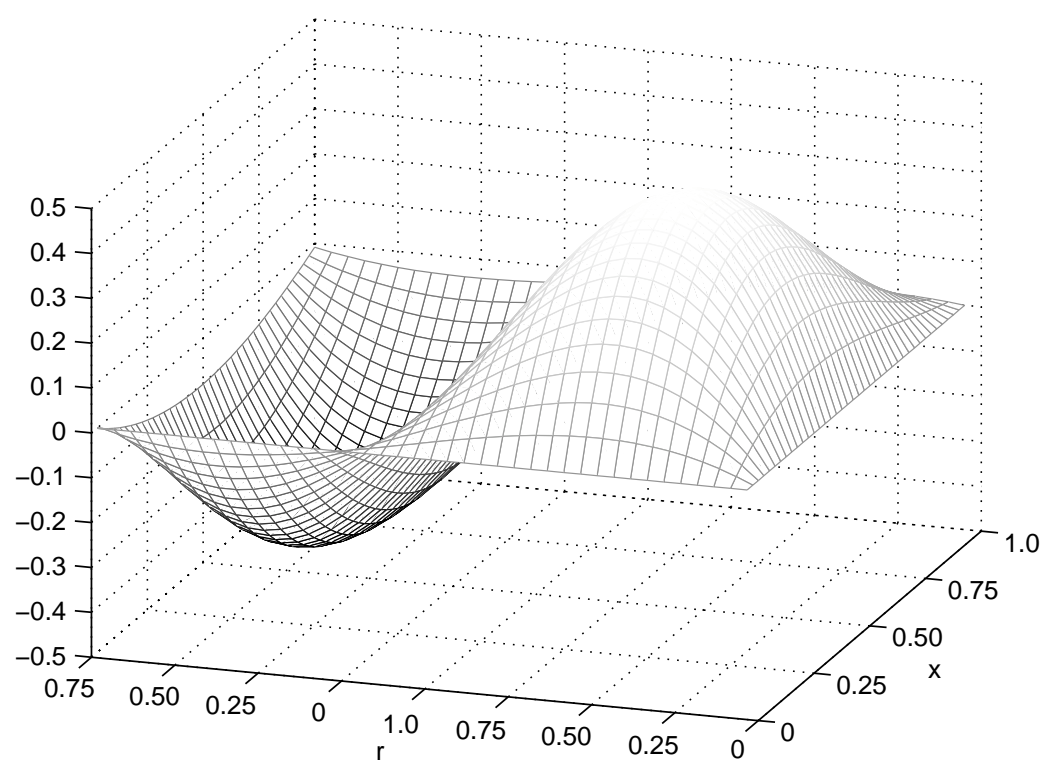

Figure 15 The difference between the initial and given PDF 


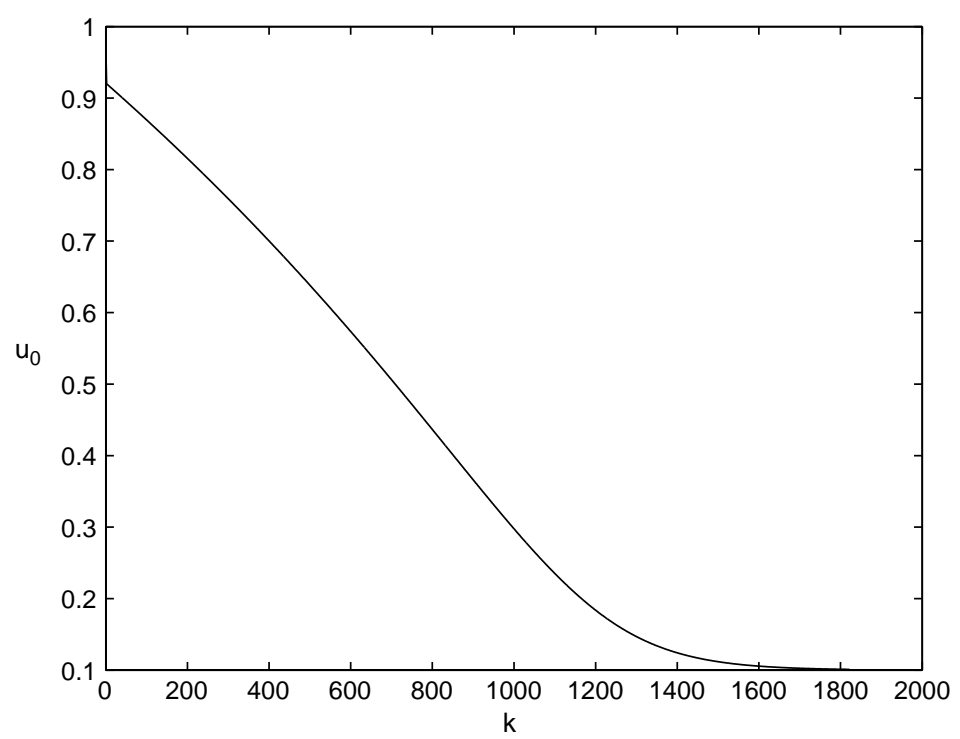

Figure 16 The control sequence of 3D static control simulation

the pre-condition for choosing the $2 \mathrm{D}$ control algorithms is that the flame temperature field can be presented by its FED. However, not all the FTD can satisfy such a condition. As such, a 3D flame distribution control algorithm has been proposed, which requires a high computational cost but can present more information on the dynamical evolution of the FTD. In addition, the 3D static control method is the first

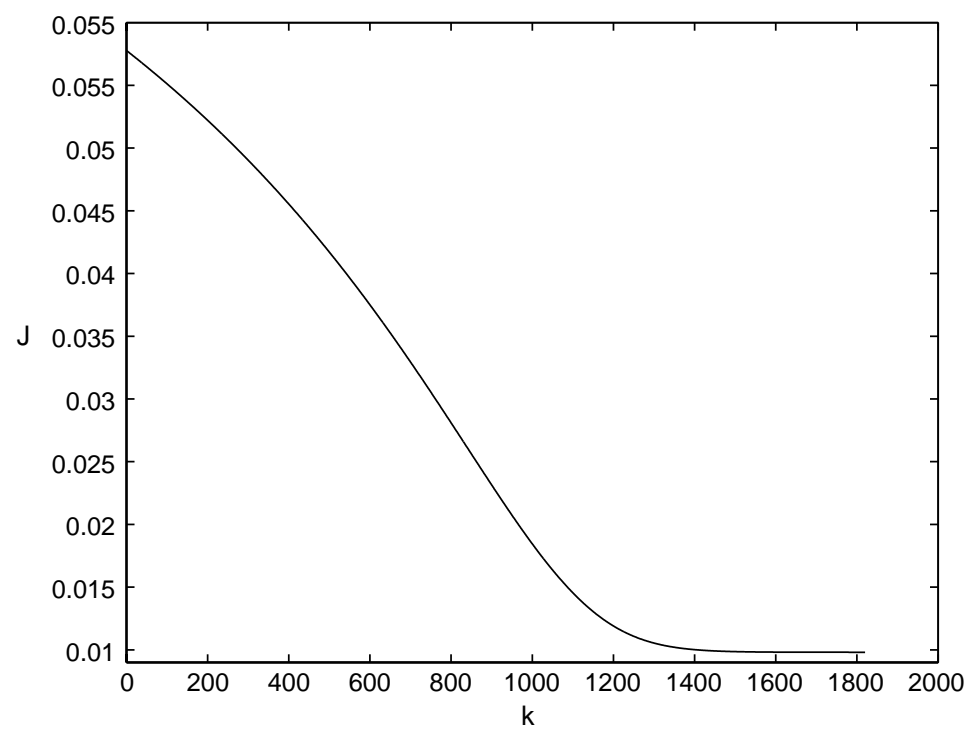

Figure 17 The performance function of 3D static control simulation 
step for the dynamic controller design of 3D irregular flame distributions. The desired results have been obtained in the simulations of these methods.

The flame distribution models for static and dynamic 2D flame control are formulated based upon the assumption that all the temperature distributions on each cross-section are similar. Such a flame model cannot be controlled by the 3D algorithm. As such, a direct comparison between the 2D and $3 \mathrm{D}$ control algorithms is not available in this paper.

\section{Acknowledgements}

The authors would like to thank the Chinese NSF grants 60534010 and 60472065 for the support of this work.

\section{References}

Dai, L. 1989: Singular control systems. SpringVerlag.

Fu, W.B., Zhang, Y.L. and Wang, Q.A. 1989: Combustion theory. Higher Education Press.

Maciejowski, J.M. 2001: Predictive control: with constrains. Prentice Hall.

Pope, S.B. 1985: PDF methods for turbulent reactive flows. Progress in Energy and Combustion Science 11, 119-92.

Sun, X.B. and Wang, H. 2004: Closed control of gas jetflames distribution using probability density function shaping techniques. In Control 2004, University of Bath, ID-088.

Sun, X.B. and Wang, H. 2005: Stable predictive control of gas jet flame distribution via the output probalility density function shaping. In Proceedings of the 13th Mediterrenean Conference on Control and Automation, Cyprus, 1458-63.

Tao, W. and Burkhardt, H. 1994: Application of fuzzy logic and neural network to the control of a flame process. In Proceedings of the Second International Conference on Intelligent Systems Engineering, Hamburg, 235-40.

Wang, H. 2000: Bounded dynamic stochastic systems: modeling and control. SpringVerlag.

Wang, H., Zhang, J.F. and Yue, H. 2005: Multistep predictive control of a PDF-shaping problem. Acta Automatica Sinica 31, 274-79.

Wang, J., Du, J. and Wang, S.Y. 1995: Study and application of energy balance method and on-line thermal efficiency. Electric Power $12,38-41$.

Yue, H. and Wang, H. 2003: Recent developments in stochastic distribution control: a review. Measurement and Control 36, 209-15.

Zhou, C.H. 1996: Model establishment of fuel controlled objective in utility boilers based on signal of radiative energy from furnaces and simulation on its control. Proceedings of the CSEE16 4, 226-29. 